universal solution, or ensured compatibility between different solutions, is needed. It is inconceivable that vaccinated people, healthcare professionals and authorities will adopt numerous EVCs simultaneously. Thus, isolated solutions in deliberate opposition to international and regional standardization efforts-such as the French government's deciding not to adopt the Apple-Google contact-tracing solution and thus forfeiting interoperability with apps in other European countries ${ }^{10}$ - need to be avoided.

To ensure that EVCs experience a faster and more coordinated rollout than contact-tracing apps did earlier during the pandemic, it would behoove policymakers and practitioners alike to take a critical look back at the standardization processes of these apps and the lessons learned. First, neither companies nor consortia or governments alone were able to set a standard. This highlights the need for coordinated multi-stakeholder collaboration in the development, propagation and adoption of a standardized EVC. However, the present case shows the complexity of actor involvement: the International Air Transport Organization, which ostensibly represents all airlines, is engaged in the development of an industry-wide EVC standard. At the same time, however, a number of leading airlines are involved in the development of an alternative solution. Second, for app-based EVCs, it might prove especially important to include the main technical platform owners, Apple and Google, as their involvement has been shown to enable the rapid rollout and adoption of contact-tracing apps through the inclusion of key functionality in their mobile operating systems. The early involvement of technological actors also ensures that potential technological barriers can be identified and rectified early in the development process to ensure frictionless rollout and operation. Third, government and industry decision-makers need to be aware of the importance of diffusing a universal standard, rather than prioritizing short-term national or commercial interests. Once a common standard is set, the role of national governments and industry is to provide linkages with national health infrastructure and localized user interfaces and to encourage widespread adoption of EVCs.

In conclusion, EVCs seem to be a promising technology for supporting the resumption of activities suspended by COVID-19, once the ethical concerns and implications surrounding them have been sufficiently deliberated. However, for full capture of the utility of this technology in practice, the important issue of setting a universal standard cannot be understated. In the current early stage of development, there is still a chance to homogenize solutions and prevent unnecessary delays due to the competition between and incompatibility of various solutions.

\section{Klaus Marhold (iD) $1 \bowtie$ and Jan Fell (iD) 1,2}

${ }^{I}$ Institute for Entrepreneurship and Innovation, Vienna University of Economics and Business,

Vienna, Austria. ${ }^{2}$ Institute of Service Science, National Tsing Hua University, Hsinchu, Taiwan.

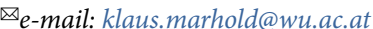

Published online: 4 March 2021

https://doi.org/10.1038/s41591-021-01286-w

References

1. Eisenstadt, M., Ramachandran, M., Chowdhury, N., Third, A. \& Domingue, J. IEEE Open J. Eng. Med. Biol. 1, 148-155 (2020).

2. Wilson, K., Atkinson, K. M. \& Bell, C. P. Am. J. Trop. Med. Hyg. 94, 485-488 (2016).

3. Singer, N. The New York Times https://www.nytimes.com/2020/ 12/13/technology/coronavirus-vaccine-apps.html (2020).

4. Voo, T. C., Clapham, H. \& Tam, C. C. J. Infect. Dis. 222, 715-718 (2020)

5. Liew, C.H. \& Flaherty, G.T. J. Public Health https://doi. org/10.1093/pubmed/fdaal25 (2020).

6. Vota, W. ICTworks https://www.ictworks.org/ digital-yellow-card-covid-19-immunization/ (2020).

World Health Organization. https://www.who.int/news-room/ articles-detail/world-health-organization-open-call-fornomination-of-experts-to-contribute-to-the-smart-vaccinationcertificate-technical-specifications-and-standards-applicationdeadline-14-december-2020 (2020).

8. International Air Transport Association. https://www.iata.org/en/ programs/passenger/travel-pass/ (2020).

9. Marhold, K. \& Fell, J. SSRN https://doi.org/10.2139/ssrn.3598143 (2020).

10. Khan, M. \& Abboud, L. The Financial Times https://www.ft.com/ content/7416269b-0477-4a29-815d-7e4ee8100c10 (2020).

\title{
The battle for COVID-19 vaccines highlights the need for a new global governance mechanism
}

To the Editor-Although the rapid development of several vaccines against COVID-19 is an unparalleled scientific accomplishment, one made possible through the collaboration of researchers, industry and funding bodies, the absence of a system that secures equitable access to vaccines has uncovered deep fissures in the global governance systems for health, as noted in a recent Nature Medicine Editorial ${ }^{1}$.

For example, advance purchase agreements for vaccines against COVID-19 have favored affluent countries, allowing them to secure $150-500 \%$ of their predicted needs ${ }^{2}$, while many citizens of low-and middle-income countries (LMICs) will remain unvaccinated until 2024.

Additionally, the power of patent-holders and pharmaceutical companies to place conditions on the use of vaccines prices out access for LMICs, and bilateral purchasing deals are rarely disclosed.

By affording priority on the basis of economic or political power, today's discourse clearly deviates from previous ethical and public-health principles of maximizing lives or life-years saved, and the sentiment that "people's entitlement to lifesaving resources should not depend on nationality" 3 .

The COVID-19 pandemic has tested wealthy nations' commitments to Agenda 2030 (ref. ${ }^{4}$ ) and to 'leaving no one behind' at the same time that it has revealed democratic deficits, institutional rigidity, weak accountability systems, and inadequate policy space that protects health-governance systems from economic goals ${ }^{5}$.
Thus, the as-yet-limited support for the vaccine-sharing and allocation principles of the COVAX initiative ${ }^{6}$ may be a sign not only of a moral catastrophe, to quote the director-general of the World Health Organization (WHO), but also of inadequate global accountability mechanisms that exposes the consequences of commercial determinants of health.

The ongoing battle for scarce vaccines against COVID-19 also highlights the lack of legally binding mechanisms that hold market actors accountable for failing to act for the public good, and the absence of global mechanisms for coordinating the pooling and sharing of resources.

In a recent example, high-income countries blocked an effort to enable timely, affordable access to products for 
the 'prevention, containment or treatment' of COVID-19, when India and South Africa proposed that the World Trade Organization temporarily suspend Trade Related Aspects of Intellectual Property Rights rules ${ }^{7}$.

Already in 2019, a Lancet Commission recommended increased utilization of legal mechanisms to advance global health through justice systems ${ }^{8}$ and to harness the power of law to promote global health and sustainable development, calling upon governments, the WHO and United Nations organizations to make necessary legal reforms to translate vision into action.

The current crisis is a salient opportunity for strengthening the global response to upcoming pandemics. With the belief that vaccines against COVID-19 are a global common good and that allocation should be based on need, we make the following recommendations.

First, a global legal framework is needed to regulate the commercial determinants of health and to secure universal fair access to essential technologies and vaccine production. This may be financed through existing alliances (such as COVAX, or Gavi, the Vaccine Alliance) or by new G7 or G20 standing funds for vaccine development and allocation ${ }^{9}$. In times of emergency, the World Trade Organization should also grant temporary waivers for Trade Related Aspects of Intellectual Property Rights rules for governments to produce their own vaccines or, if necessary, to exercise compulsory licensing powers for a third party to produce a patented technology. Ideally, perceived legal risk could facilitate the negotiation of purchase transparency, voluntary licensing arrangements and patent sharing while committing the industry to global justice.
Second, to strengthen crisis preparedness $^{4}$ and make the response less blunt and more evidence-based, coordinated and effective, the existing gaps in infrastructure, technology and information must be reduced. The WHO warns that lockdown-induced disruption (e.g., of childhood immunization and essential care) could cause greater death and suffering than COVID-19 itself $^{10}$. As LMICs embark on an unprecedented vaccination scale-up, substantial investment is needed to prevent redistribution of scarce health personnel that further undermines routine immunization. Future plans must have a clear health-systems dimension and must include social mobilization and strategies for fighting vaccine misinformation? .

Third, as shown by COVID-19, updated global governance mechanisms are needed that better reflect the contemporary geopolitical order and truly encourage international collaboration across sectors, through political and legal solutions rooted in commitments to justice and shared global responsibility ${ }^{5,8}$. We call for a 21st-century Bretton Woods-type conference, similar to that of 1944, when states met to improve global economic interaction, secure peace and provide assistance to countries devastated by World War II, which resulted in new global governance structures such las the International Monetary Fund and the World Bank.

Using the dynamics of one acute crisis to build resilience against the next could also serve a greater good in line with the growing notion of the United Nations Security Council that health, poverty mitigation, security and peace are interlinked.
Anna Mia Ekström (D) 1 , Camilla Berggren², Göran Tomson ${ }^{2,3}$, Lawrence O. Gostin ${ }^{4}$, Peter Friberg 2,5 and Ole Petter Ottersen ${ }^{3}$ ${ }^{1}$ Department of Global Public Health, Karolinska Institutet, Stockholm, Sweden. ${ }^{2}$ Swedish Institute for Global Health Transformation, Royal Swedish Academy of Sciences, Stockholm, Sweden. ${ }^{3}$ President's Office, Karolinska Institutet, Stockholm, Sweden. ${ }^{4} O$ 'Neill Institute for National and Global Health Law, Georgetown University, Washington, DC, USA.

${ }^{5}$ Department of Public Health and Community medicine, Sahlgrenska Academy, Gothenburg University, Gothenburg, Sweden.

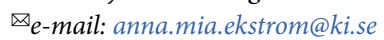

Published online: 11 March 2021

https://doi.org/10.1038/s41591-021-01288-8

References

1. Nat. Med. 27, 1 (2021).

2. Launch \& Scale Speedometer. https://launchandscalefaster.org/ COVID-19 (2021)

3. Emanuel, E. J. et al. Science 369, 1309-1312 (2020).

4. Ottersen, O. P. \& Engebretsen, E. Nat. Med. 26, 1672-1673 (2020).

5. Ottersen, O. P. et al. Lancet 383, 630-667 (2014).

6. COVAX. Gavi, the Vaccine Alliance https://www.gavi.org/sites/ default/files/covid/covax/COVAX_Principles-COVID-19Vaccine-Doses-COVAX.pdf (2020).

7. Deutsche Welle. https://www.dw.com/en/rich-countries-blockindia-south-africas-bid-to-ban-covid-vaccine-patents/a56460175 (2021)

8. Gostin, L. O. et al. Lancet 393, 1857-1910 (2019).

9. Gostin, L.O., Friedman, E.A. \& Moon, S. Foreign Affairs https://www.foreignaffairs.com/articles/world/2021-01-19/ wealthy-countries-should-share-vaccine-doses-it-too-late (2021)

10. World Health Organization. https://www.who.int/news/item/ 15-07-2020-who-and-unicef-warn-of-a-decline-in-vaccinationsduring-covid-19(2020)

Author contributions

All authors contributed to the writing of this manuscript; A.M.E. drafted the first version and edited the final version on the basis of feedback from all co-authors; and all authors have seen and approved the submitted version.

\section{Comprehensive large-scale nucleic acid- testing strategies support China's sustained containment of COVID-19}

To the Editor-Since identification and initial characterization of COVID-19 in late December 2019 and early January 2020, China's containment goal has been complete interruption of local transmission of the coronavirus SARS-CoV- $2^{1}$. The nationwide epidemic wave centered on Wuhan was contained by April 2020 with non-pharmaceutical interventions alone but was associated with economic loss: China's first-quarter gross domestic product fell $6.8 \%$ compared with that in the first quarter of 2019 (ref. ${ }^{2}$ ). Since containment, there have been numerous outbreaks in China caused by imported viruses with local spread, varying in size from several cases to about 1,000 cases, all stopped with non-pharmaceutical interventions alone $\mathrm{e}^{3}$. Sustained containment has high socioeconomic value: economic activities recovered rapidly, with an increase in gross domestic product of $3.2 \%$ during April through June 2020, and of 4.9\% during July through September 2020 ( ref. $^{2}$ ); the government's response to the pandemic was perceived positively by the Chinese public ${ }^{4}$. In this Correspondence, we describe two large-scale PCR-based testing strategies that 